\title{
Comparative Study for Carrot Juice and Selenium Supplement in Many Physiological and Biochemical Parameters in Patients with Rheumatoid Arthritis in Kirkuk City
}

Wedad Mahmood Lahmood Al-obaidi ${ }^{1}$ Mohanad Hasan Mahmood Al-Izzi², Aya Saad yaseen²

DOI. 10.21931/RB/2021.06.04.15

Abstract: Carrot juice is a critical source of vitamins, selenium, and $\beta$-carotene, which is suggested to protect from Rheumatoid Arthritis (RA). The present study aimed to show the effect of carrot juice supplementation compared to selenium tablet supplementation, so our study includes (44) blood samples belonging to young men with RA. All patients aged (20-45) years, 44 blood samples were obtained before treatment at week (0), twenty-four men with RA were supplied with fresh carrot juice. The other group of 20 patients was given an artificial selenium supplement for 21 days as well, and the results were analyzed. The samples were collected from Kirkuk hospital, and external specialized clinical from October/2019 to September /2020; experimental groups were divided into three groups: Group 1 : (44) men Rheumatoid arthritis (RA) before treatment, Group 2: (24) men with RA+ Carrot juice, Group 3: (20) men with RA + Selenium Tab, We reach to following results: the patients who have RA consumption Carrot juice and patients take up Se tab. show significant decrease respectively in RBCs, WBCs, ERS, and RF compared with the Rheumatoid arthritis group, so as the results show a significant decrease in Leptin, IL-6, C-Reactive Protein, and TNF-a concentrations in comparison with the Rheumatoid arthritis group. In contrast, we found a significant increase in GSH, Selenium concentrations, and VD3 in men with RA administration carrot juice and patients' consumption Se tab. Respectively compared with the RA group and, finally, our finding shows no difference in Ceruloplasmin in experimental groups.

Key words: Rheumatoid Arthritis, Carrot Juice, antioxidant agents, Selenium,VitD3, and Leptin.

\section{Introduction}

Rheumatoid arthritis (RA) is an autoimmune, chronic, and inflammatory disease that affects $\sim 1 \%$ of the global population $^{1}$. It is characterized by excessive production of inflammatory mediators, including cytokines (IL-1ß, IL-6, TNF-a, IL-17), chemokines, and autoantibodies that lead to an exacerbated activation of immune system cells. If not treated promptly and adequately, this disease can lead to irreversible joint damage 2. it has been considered a multifactorial disease resulting from the interaction of genetic, hormonal, and environmental factors that contribute to the loss of immune tolerance ${ }^{3-5}$. Among the environmental risk factors, the diet has been reported to play an essential role in regulating RA disease activity as specific foods can aggravate or attenuate inflammation ${ }^{6}$. Recently, it has been established that nutrition is essential for the proper development of the immune system, which has led to the study of the relationship between both of them ${ }^{7}$.

Fruits and vegetables are rich sources of nutrients that contain phytochemicals (also known as bioactive compounds), which are recognized for their nutraceutical effects and health benefits. The cultivated carrot (Daucus carota L.) is one of the most essential vegetable plants globally because of its high yield potential and use as a fresh or processed product ${ }^{8}$. Although pharmacological treatment of RA patients is currently more effective, not all treatments achieve the reduction of the disease but immune-nutrients in the diet of RA patients could be an alternative to improve some disease conditions such as pain alleviation, reduced count of tender joints, and shortening of the morning stiffness duration, which can also influence on the attenuation of the disease clinical activity ${ }^{9}$. The importance of the adequate consumption of a diet rich in micronutrients is that they have a fundamental role in the immune system throughout life. Among the micronutrients, vitamins A, C, D, $E$, and B12 and minerals such as iron, zinc, and selenium are involved in activating and functioning the immune response $\mathrm{e}^{10,11}$

Selenium (Se) is an essential micronutrient that is important for various aspects of human health, including proper thyroid hormone metabolism, cardiovascular health, prevention of neurodegeneration and cancer, and optimal immune responses. Very low (depleted) or very high (toxic) levels of Se intake can be detrimental or possibly fatal. Extreme deficiency or toxicity is not commonly found in humans, but selenosis has been reported in cases of miscalculated supplement formulations, suicides, accidental overdose, or intentional poisoning ${ }^{12}$. That said, less overt changes in Se status within an individual may still affect inflammation and immune responses. The biological effects of Se are mainly exerted through its incorporation into selenoproteins, and selenoproteins are involved in the activation, proliferation, and differentiation of cells that drive innate and adaptive immune responses. Dietary Se and selenoproteins are not only important for initiating or enhancing immunity but are also involved in immunoregulation, which is crucial for preventing excessive responses that may lead to autoimmunity or chronic inflammation. It should be noted that most studies in the literature involve modifications to dietary Se, and insights into mechanisms often are not clear, but roles for individual selenoproteins and mechanisms are discussed when data are available.

Leptin is a cytokine-like $16 \mathrm{kDa}$ peptide produced mainly through adipose tissue and regulates food intake, basal metabolism, and the $\beta$-oxidation of fatty acids. When binding to its receptor(s) located in hypothalamic nuclei occurs, leptin is an essential trigger of adaptive mechanisms during starvation leading to downregulation of thyroid and reproductive functions and stimulation of the hypothalamus. In healthy subjects, leptin levels in the blood are proportional to the body fat mass. Leptin has recently been recognized as a modulator of inflam-

${ }^{1}$ Department of Biology-College of Education-Al-Hawija University of Kirkuk.

${ }^{2}$ Department of Biology- College of Science- University of Tikrit. 


\section{Pathogenesis of Rheumatoid Arthritis}

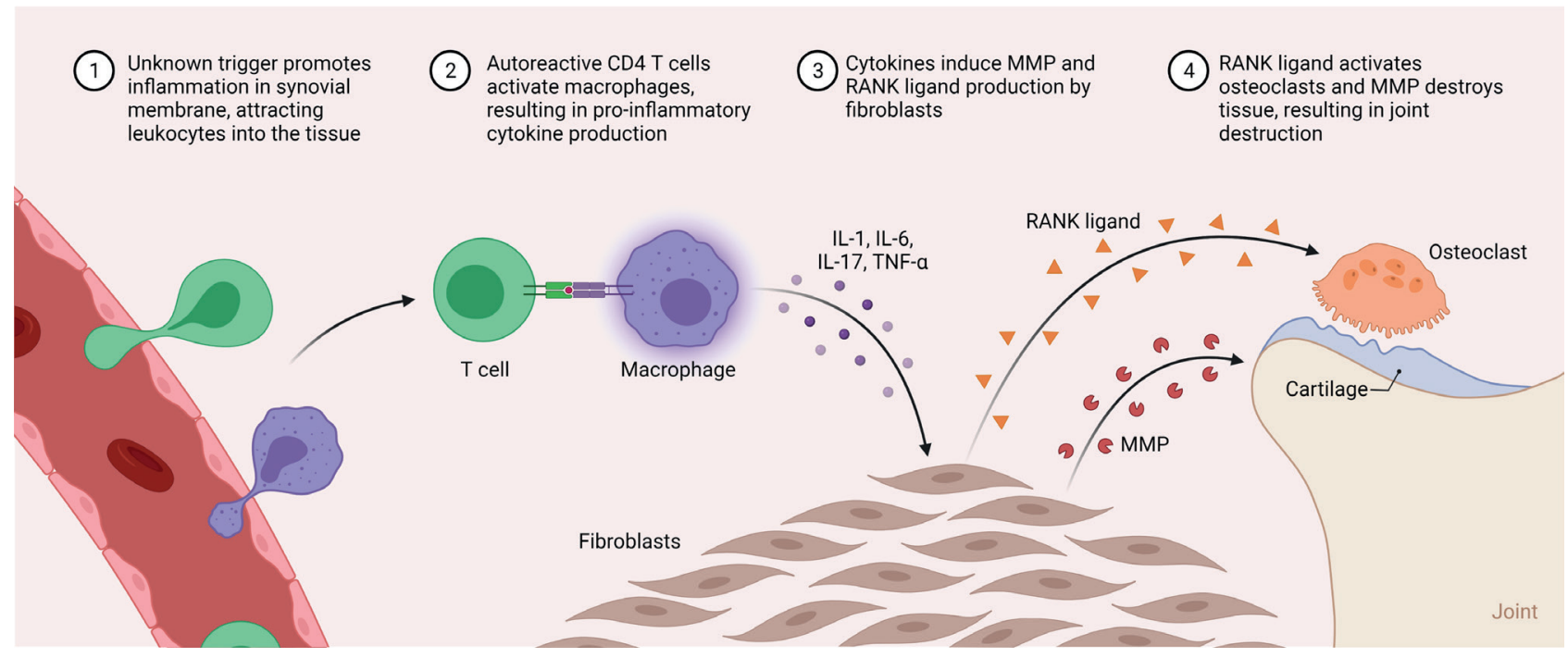

Figure 1. Rheumatoid arthritis (RA) is an autoimmune, chronic, and inflammatory disease. It is characterized by excessive production of inflammatory mediators, including cytokines (IL-1ß, IL-6, TNF-a, IL-17), chemokines, and autoantibodies that lead to an exacerbated activation of immune system cells.

matory and immune responses. Indeed, leptin participates in bone formation by stimulating osteoblastic cell proliferation and the formation of mineralized nodules in primary osteoblasts and osteosarcoma cells. Leptin facilitates the proliferation of human endothelial cells supporting angiogenesis and neovascularization. Leptin has a dual role in inflammation.

On the one hand, it activates monocyte/macrophage cells and potentiates the production of the pro-inflammatory cytokines, tumor necrosis factor a (TNFa), interleukin (IL)6, and directs $T$ cell differentiation to Th1 phenotype, expressing interferon $\mathrm{Y}^{13}$. Vitamin $\mathrm{D}$ is a secosteroid hormone involved in bone and calcium metabolism. It is involved in regulating calcium homeostasis, as it regulates calcium absorption from the gastrointestinal system. The hormone is synthesized in the skin by ultraviolet irradiation Vitamin D has extraskeletal effects as well ${ }^{14}$. The non-classical actions of vitamin $\mathrm{D}$ are currently under discussion. Vitamin $\mathrm{D}$ has been found to have immunomodulatory actions; Vitamin $\mathrm{D}$ deficiency has been shown to be correlated with the appearance of autoimmune diseases, such as diabetes mellitus type 1 and multiple sclerosis; Vitamin D deficiency may increase the risk for the development of $\mathrm{RA}^{15}$. Recently, the role of vitamin $\mathrm{D}$ deficiency in the pathogenesis of RA and the relationship between vitamin $D$ deficiency and the activity of RA is discussed. RA is an inflammatory disease characterized by flares and remissions, flares being characterized by pain. Vitamin $D$ deficiency is also known to be associated with diffuse musculoskeletal pain ${ }^{16}$.

The current study aims to clarify the effect of Carrot juice administration rich with vitamins, antioxidants, and minerals as selenium to treat Rheumatoid arthritis in men in comparison with artificial selenium supplement main role in increasing the production and release of the antioxidants and minerals and reducing inflammation factor.

The study also aims to elucidate the role of natural components for carrots in improving human bone and cartilage abilities through its effect as a natural inducer, dispensing as much as possible with medication or excessive treatment without consulting a doctor. This is because these supplements

are an essential natural requirement for the human body in general and particularly for patients in Kirkuk city.

\section{Methods}

\section{Experimental Design}

Our study include (44) blood samples belonging to young men with RA, we withdraw (44) blood samples before treatment in a week $(0)$, twenty-four men with RA volunteered to consumption fresh carrot juice rich naturally with antioxidants agents and minerals (Fe and Selenium ) for twenty-one days, while the other (20) volunteered has taken selenium tablets as an artificial supplement, With age (20-45) year, the samples were collected from Kirkuk hospital and clinical from October /2019 to September /2020 the samples were given within a specific measurement and a regular schedule at a rate of $250 \mathrm{ml}$ per sample in Every morning for 21 days. experimental groups were divided into three groups: Group 1 : (44) men Rheumatoid arthritis(RA) before treatment, Group 2: (24) men with RA+ Carrot juice, Group 3: (20) men with RA +Selenium Tablet.

The beginning fasting was done for the samples for 8 hours, after which blood samples were drawn through the vein in the arm with the use of the tanka to show the vein wall; the blood was placed in Test tubes and placed in the incubator at a temperature of $37^{\circ}$ for 30 minutes, after which a centrifuge was used Central at a speed of 3000 cycles per minute duration is 15 minutes. Separate the serum from the other ingredients using a micropipette and place it at a temperature of $-20^{\circ} \mathrm{C}$ until the Physiological and biochemical tests are performed.

\section{Determination of parameters}

Estimation total number of RBCs, WBCs, the results were calculated automatically by Hematology Analyzer. In contrast, the results of Erythrocytes sedimentation rate (ESR) calcu- 
lated by Westergren method is considered the standard method for measuring ESR as a nonspecific indicator of disease activity. Clinicians often use it in assisting the diagnosis and follow-up of many inflammatory disorders ${ }^{17}$. Rheumatoid factor (RF) the most commonly used serological method is the latex agglutination test. RF is an IgM class of antibody directed against the Fc portion of the $\lg G$ molecule; it is detected by its ability to agglutinate the latex particles coated with the $\lg G$ molecule.

Estimation of antioxidant agents -Selenium concentration in blood serum ${ }^{18}$ estimation Glutathione and Ceruloplasmin, Estimation of VitD3 Concentration in blood serum for Experimental groups: the assay principle combines enzyme immunoassay competition method with a final fluorescent detection ELFA (Enzyme-Linked Fluorescent Assay) technique by using mini vidas device ${ }^{19}$.

Estimation of Leptin concentration, IL-6 , C-Reactive Protein and TNF-a level in blood serum.

\section{Statistical analysis}

The one-way analysis of variance (ANOVA) is used to determine whether there are any statistically significant differences between the means of two or more groups. The arithmetic averages of the coefficients were tested using the Dunkin' multiple range test with a level of significance (0.05) to determine the significant differences between the groups.

Results

Effect of consumption Carrot juice in RBCs, WBCS, ESR, and Rheumatoid factor (RF)in Patients with Rheumatoid Arthritis

Our study shows a significant decrease in RBCs count in patients with Rheumatoid arthritis after consuming fresh carrot juice and selenium group 4.0250, 4.5120 respectively in comparison with Rheumatoid arthritis without treatment 6.7962. The results proved that in Table (1) the WBCs was high to 14100 we reduced it to $9171.2,11660$ in groups 2 \& 3 So as the ESR also decreased from 43.212 to 29.295 , 33.0114 respectively. Our study shows a significant decrease in RF level in patients with Rheumatoid arthritis after consuming fresh carrot juice $6.4011,6.4482$ respectively, compared with the Rheumatoid arthritis group 8.4963

\section{Effect of consumption Carrot juice in Leptin, IL-6, C-Reactive} Protein and TNF-a in Patients with Rheumatoid Arthritis

Our study shows a positive effect for consuming fresh carrot juice in leptin concentration in men suffering from Rheumatoid Arthritis, so our study shows a significant decrease in Leptin level In patients with RA after consuming fresh carrot juice Selenium tab. 23.550, 43.440. Respectively compared with RA with out treatment 56.111 , Our study shows a significant decrease in IL-6 concentration in patients with Rheumatoid arthritis after consuming fresh carrot juice and selenium group 2.22 \&4.22 respectively compared with Rheumatoid arthritis without treatment 9.67. Our study shows a significant decrease in CRP in patients with Rheumatoid arthritis after consuming fresh carrot juice and selenium group 2.10 \& 2.05 respectively compared with Rheumatoid arthritis without treatment 8.73. At the same time, TNF-a concentration witnessed a high decrease in patients with Rheumatoid arthritis after consuming fresh carrot juice and selenium group $20.55 \&$ 25.2.77 respectively compared with Rheumatoid arthritis without treatment 48.53

\section{Effect of consumption Carrot juice in Selenium, Glutathione Ceruloplasmin and VitD3 in Patients with Rheumatoid arthritis}

The recent result in our study in table 3 shows a highly significant increase in Selenium (Se) concentration in patients $\mathrm{RA}+$ Selenium tablets group, and in RA + carrot juice 86.0021, 66.5400 respectively in compare with Rheumatoid Arthritis without treatment 26.5647 As well as Glutathione(GSH) concentration observed a significant increase in patients with Rheumatoid arthritis consume fresh carrot juice and in RA+ Se tab. Group respectively 3.902, 3.013 as compared with Rheumatoid arthritis without treatment 1.011 So as in VitD3 in patients with Rheumatoid arthritis consume Se tab. And in patients RA +carrot juice $77.0141,74.2907$ respectively as compared with Rheumatoid arthritis without treatment 19.831 While Ceruloplasmin concentration did not have any change in our experimental groups as shown in Tablet 3 .

\begin{tabular}{|lllll|}
\hline Parameters Group & RBCs cells/mcL & WBCs Cells/mcL & ESR mm/hr & RF IU/ml \\
\hline Rheumatoid group & $8.4963 \pm 0.6046$ & $43.212 \pm 19.9770$ & $14100 \pm 32.6390$ & $6.7962 \pm 0.1321$ \\
\hline $\begin{array}{l}\text { Rheumatoid } \\
\text { group+Carrot juice }\end{array}$ & $6.4482 \pm 0.4542$ & $29.295 \pm 7.00890$ & $9171.2 \pm 14.4271$ & $4.0250 \pm 0.1159$ \\
\hline $\begin{array}{l}\text { Rheumatoid group + } \\
\text { Selenium tab. }\end{array}$ & $6.4011 \pm 0.40110$ & $33.0114 \pm 10.1210$ & $11660 \pm 16.0022$ & $4.5120 \pm 0.1200$ \\
\hline
\end{tabular}

Table 1. The concentration of RBCs, WBCS, ESR, and Rheumatoid factor (RF) in experimental groups.

\begin{tabular}{|lcllc|}
\hline Parameters Group & TNF- $\alpha \mathrm{pg} / \mathrm{ml}$ & $\begin{array}{l}\text { C-reactive protein } \\
\mathrm{mg} / \mathrm{dl}\end{array}$ & IL-6pg/ml & Leptin $\mathrm{ng} / \mathrm{dl}$ \\
\hline Rheumatoid group & $48.53 \pm 7.00$ & $8.73 \pm 1.22$ & $9.67 \pm 2.28$ & $56.111 \pm 17.44$ \\
\hline $\begin{array}{l}\text { Rheumatoid } \\
\text { group+Carrot juice }\end{array}$ & $20.55 \pm 2.052$ & $2.10 \pm 1.00$ & $2.22 \pm 0.15$ & $23.550 \pm 7.500$ \\
\hline $\begin{array}{l}\text { Rheumatoid group }+ \\
\text { Selenium tab. }\end{array}$ & $25.2 .77 \pm 2.55$ & $2.05 \pm 1.24$ & $4.22 \pm 2.11$ & $43.440 \pm 12.22$ \\
\hline
\end{tabular}

Table 2. The concentration of Leptin, IL-6, C-reactive Protein, and TNF-a in experimental groups. 


\begin{tabular}{|c|c|c|c|c|}
\hline Parameters Group & VitD3 IU/L & $\begin{array}{l}\text { Ceruloplasmin } \\
\mu \mathrm{mo} / \mathrm{L}\end{array}$ & $\begin{array}{l}\text { Glutathione } \\
\text { Mmo/L }\end{array}$ & Selenium ng/ml \\
\hline Rheumatoid group & $19.831 \pm 8.7321$ & $195.95 \pm 2.88$ & $1.011 \pm 0.370$ & $26.5647 \pm 11.0467$ \\
\hline $\begin{array}{l}\text { Rheumatoid group + } \\
\text { Carrot juice }\end{array}$ & $74.2907 \pm 14.564$ & $209.36 \pm 2.92$ & $3.902 \pm 0.813$ & $66.5400 \pm 15.7306$ \\
\hline $\begin{array}{l}\text { Rheumatoid group }+ \\
\text { Selenium tab. }\end{array}$ & $77.0141 \pm 14.591$ & $196.16 \pm 2.81$ & $3.013 \pm 0.800$ & $86.0021 \pm 11.0122$ \\
\hline
\end{tabular}

Table 3. The concentration of Selenium, Glutathione, Ceruloplasmin, and VitD3, in experimental groups.

\section{Discussion}

Our study found a significant decrease in RBCs, WBCs count in patients with Rheumatoid arthritis after consuming fresh carrot juice; the previous studies showed that autoimmune disorders and infections could result in a high white blood cell count, but a diet rich in vegetables can help reduce its levels reach to normal value White blood cells are important because they fight bacteria and viruses that could cause illness. A reduced count under average level may depress your immunity. A multivitamin, taken in conjunction with plenty of vegetables, can help you get the recommended daily intake of white blood cell-boosting nutrients. On a cellular level, dietary may influence various leukocytic effector functions, including adherence, migration, phagocytosis, and cytokine secretion. Several members of the selenoprotein family regulate or are regulated by cellular redox tone, a crucial modulator of immune cell signaling and function ${ }^{20}$. So our study agreed with Shweta, and he follows (2017) they found that a fasting of 7-10 days with the partial nutrient intake of vegetable broth, herbal teas, parsley, garlic, and decoction of juice extracts from carrots, celery; and a controlled daily energy intake followed by 1 year of a vegan diet as compared to omnivorous diet was studied in different trials, Together these studies observed a remarkable decrease in swollen and tender joints, pain, erythrocyte sedimentation rate (ESR), and C-reactive protein ${ }^{21}$.

Dietary selenium (Se), mainly through its incorporation into selenoproteins, plays an essential role in inflammation and immunity. Adequate levels of Se are essential for initiating immunity, but they are also involved in regulating excessive immune responses and chronic inflammation. Evidence has emerged regarding roles for individual selenoproteins in regulating inflammation and immunity, and this has provided important insight into mechanisms by which Se influences these processes. Se deficiency has long been recognized to negatively impact immune cells during activation, differentiation, and proliferation. This is related to increased oxidative stress; RA is a chronic disease requiring long-term intake of drugs, including anti-rheumatics and non-steroidal anti-inflammatory drugs.

Patients with RA are prone to drop out of drug treatment due to the adverse effects. In RA, free radicals are associated with joint inflammation and damage. Antioxidant supplements and diets have long been advocated for the treatment and prevention of RA due to their protective role against free radicals ${ }^{22}$, it is well-established the role of leptin as a growth factor for the monocytes, promoting phagocytic function and proliferation of circulating monocytes, inducing the production of pro-inflammatory cytokines (TNF-a, IL-6, and IL-12) and stimulating the oxidative burst as well as the chemotactic responses mediating the inflammatory infiltrate. On the other hand, ROS production in HIV-infected patients indicates pro- grammed cell death in monocytes. Even though vitamins and selenium with antioxidant properties have been demonstrated to be beneficial to RA in a cellular study, there are contradicting results concerning the effects of antioxidant vitamins on the development of RA in animal and clinical studies. In the present study, the effects of selenium on the inflammatory cytokine networks were observed. When treated with a diet rich with selenium compared with selenium supplement, the levels of leptin, TNF-a, and IL-6 were significantly reduced in addition to reduce the C-RP and TNF-a levels of were significantly reduced increased. This suggests that vitamins suppress the inflammatory reaction in RA by increasing the levels of anti-inflammatory cytokines and reducing inflammatory cytokines. Due to the complicated cytokine networks in RA, cytokines interact by several signal transduction pathways. Therefore, the simulative effects of different vitamins and trace elements on inflammatory factors levels may be counteracted by the interaction between cytokines in $\mathrm{RA}^{23,24}$.

Our study agreed with the previous study, showing that antioxidant agents were significantly higher in the selenium-treated sheep than in control in the samples taken 14 days after lambing and 30 days after lambing ${ }^{25,26}$. Another paper showed that treatments of Selenium supplementation affected an increase in whole blood antioxidant agents and antioxidant enzyme glutathione peroxidase (GSH-Px) as well as increased plasma Selenium(Se) concentrations in experimental groups. On the other hand, the liver Se exhibited a dose-response relationship to treatment, but kidney Se concentrations were unaffected by treatment. Some dietary meat rich with Se concentrations can therefore be increased by supplementation and could contribute to increased human dietary intakes of the element ${ }^{27}$, as well as carrot juice good source of carotenoids which act as antioxidants and help detoxify the system because antioxidants fight free radicals and reduce oxidative stress so they help stimulate metabolism ${ }^{28,29}$. The data support a role for vitamin $\mathrm{D}$ deficiency in the development and progression of autoimmune inflammatory conditions in general, particularly RA. Earlier animal models indicate that the $1,25(\mathrm{OH})_{2} \mathrm{D}_{3}$ metabolite and its analogs may suppress collagen-induced arthritis. Other data suggest that vitamin $D$ receptor agonists may also prevent and suppress established collagen-induced arthritis; however, data show that vitamin $\mathrm{D}$ may be negatively affected in acute response, that is, its levels may decrease in the setting of inflammation, such as inactive RA. ${ }^{30-32}$.

Despite that, treatment with rituximab in RA did not affect vitamin D levels. However, it decreased indices of inflammation Supplementation with selenium induces a level of vitamin $D$ in plasma blood which has been proposed to induce immune tolerance and thus prevent the development of autoimmune di- 
seases $^{33}$. Recently, the combination of anti-rheumatic and the drugs of dietary supplement of selenium induce and increase the level of vitamin D has been suggested for RA Patients with RA are prone to osteoporosis as well as for its possible effects on disease activity ${ }^{34,35}$.

\section{Conclusions}

Consumption of natural juice rich with selenium, vitamin $D$, and several antioxidants agents more beneficial in the treatment of Rheumatoid arthritis than taken artificial supplements should be taken with caution given its possible toxic effects that exceed its recommended consumption limits.

\section{Conflict of Interests}

The authors of this paper declare that it has no financial or personal relationships with individuals or organizations that would change unacceptably bias the content of this paper and therefore declare that there is no conflict of interests.

\section{Bibliographic references}

1. Taylor PC. Update on the diagnosis and management of early rheumatoid arthritis. Clin Med (Lond). $2020 ; 20(6): 561-564$.

2. Smolen JS, Aletaha D, Barton A, Burmester GR, Emery P, Firestein GS, Kavanaugh A, Mclnnes IB, Solomon DH, Strand V, Yamamoto K. Rheumatoid arthritis. Nat Rev Dis Primers. 2018 Feb 8;4:18001.

3. Firestein GS, Mclnnes IB. Immunopathogenesis of Rheumatoid Arthritis. Immunity. 2017 Feb 21;46(2):183-196.

4. Alpízar-Rodríguez D, Pluchino N, Canny G, Gabay C, Finckh A. The role of female hormonal factors in the development of rheumatoid arthritis. Rheumatology (Oxford). 2017 Aug 1;56(8):1254-1263.

5. Xu B, Lin J. Characteristics and risk factors of rheumatoid arthritis in the United States: an NHANES analysis. PeerJ. 2017 Nov 24;5:e4035.

6. Skoczyńska M, Świerkot J. The role of diet in rheumatoid arthritis. Reumatologia. 2018;56(4):259-267.

7. Zapatera B, Prados A, Gómez-Martínez S, Marcos A. Immunonutrition: methodology and applications. Nutr Hosp. 2015 Feb 26;31 Suppl 3:145-54.

8. Wang L, Wang FS, Gershwin ME. Human autoimmune diseases: a comprehensive update. J Intern Med. 2015 Oct;278(4):369-95.

9. Dawid C, Dunemann F, Schwab W, Nothnagel T, Hofmann T. Bioactive $\mathrm{C}_{17}$-Polyacetylenes in Carrots (Daucus carota L.): Current Knowledge and Future Perspectives. J Agric Food Chem. 2018 Oct 28;63(42):9211-22.

10. Reedy J, Lerman JL, Krebs-Smith SM, Kirkpatrick SI, Pannucci TE, Wilson MM, Subar AF, Kahle LL, Tooze JA. Evaluation of the Healthy Eating Index-2015. J Acad Nutr Diet. 2018 Sep;118(9):1622-1633.

11. Kirkpatrick SI, Pannucci TE, Wilson MM, Subar AF, Kahle LL, Tooze JA. Evaluation of the Healthy Eating Index-2015. J Acad Nutr Diet. 2018 Sep;118(9):1622-1633.

12. Hariharan S, Dharmaraj S. Selenium and selenoproteins: it's role in regulation of inflammation. Inflammopharmacology. 2020 Jun;28(3):667-695.

13. Pérez-Pérez A, Sánchez-Jiménez F, Vilariño-García T, Sánchez-Margalet V. Role of Leptin in Inflammation and Vice Versa. Int J Mol Sci. 2020 Aug 16;21(16):5887.

14. Hewison M. Vitamin D and immune function: autocrine, paracrine or endocrine? Scand J Clin Lab Invest Suppl. 2012;243:92-102.

15. Merlino LA, Curtis J, Mikuls TR, Cerhan JR, Criswell LA, Saag KG; lowa Women's Health Study. Vitamin D intake is inversely associated with rheumatoid arthritis: results from the lowa Women's Health Study.Arthritis Rheum.2004Jan;50(1):72-7.

16. Sirbu E, Buleu F, Tudor A, Dragan S. Vitamin D and disease activity in rheumatoid arthritis patients: a retrospective study in a Romanian cohort. Acta Biochim Pol. 2020 Jun 18;67(2):267-272.
17. Hardeman MR, Levitus M, Pelliccia A, Bouman AA. Test 1 analyser for determination of ESR. 1. Practical evaluation and comparison with the Westergren technique. Scand J Clin Lab Invest. 2010 Feb;70(1):21-5.

18. Gardiner PH, Littlejohn D, Halls DJ, Fell GS. Direct determination of selenium in human blood serum and plasma by electrothermal atomic absorption spectrometry. J Trace Elem Med Biol. 1995 Jul;9(2):74-81.

19. Holick MF. Vitamin D deficiency. N Engl J Med. 2007 Jul 19;357(3):266-81.

20.Petkova-Marinova TV, Ruseva BK, Atanasova BD.Selenium deficiency as a risk factor for development of anemia. J. Biomed. Clin. Res., 2017; 10(1) : 9-17.

21. Khanna S, Jaiswal KS, Gupta B. Managing Rheumatoid Arthritis with Dietary Interventions. Front Nutr. 2017 Nov 8;4:52.

22. Gunaydin R, Kaya T, Atay A, Olmez N, Hur A, Koseoglu M. Serum leptin levels in rheumatoid arthritis and relationship with disease activity. South Med J. 2006 Oct;99(10):1078-83.

23.Pérez-Pérez A, Sánchez-Jiménez $F$, Vilariño-García T, Sánchez-Margalet V. Role of Leptin in Inflammation and Vice Versa. Int J Mol Sci. 2020 Aug 16;21(16):5887.

24. Reseland JE, Gordeladze JO. Role of leptin in bone growth: central player or peripheral supporter? FEBS Lett. 2002 Sep 25;528(1-3):40-2.

25. Hoffmann PR, Berry MJ. The influence of selenium on immune responses. Mol Nutr Food Res. 2008 Nov;52(11):1273-80.

26. da Fonseca LJS, Nunes-Souza V, Goulart MOF, Rabelo LA. Oxidative Stress in Rheumatoid Arthritis: What the Future Might Hold regarding Novel Biomarkers and Add-On Therapies. Oxid Med Cell Longev. 2019 Dec 14;2019:753-805.

27. Molnár J, MacPherson A, Dixon J. Effect of supplementation with selenium on whole blood glutathione peroxidase activities and on plasma and tissue selenium concentrations in lambs. Biol Trace Elem Res. 1996 Dec;55(3):253-62.

28. Nakajima A, Aoki Y, Shibata Y, Sonobe M, Terajima F, Takahashi H, Saito M, Taniguchi S, Yamada M, Nakagawa K. Identification of clinical parameters associated with serum oxidative stress in patients with rheumatoid arthritis. Mod Rheumatol. 2014 Nov;24(6):926-30.

29. Smallwood MJ, Nissim A, Knight AR, Whiteman M, Haigh R, Winyard PG. Oxidative stress in autoimmune rheumatic diseases. Free Radic Biol Med. 2018 Sep;125:3-14.

30.Olejnik A, Kowalska K, Kidoń M, Czapski J, Rychlik J, Olkowicz M, Dembczyński R. Purple carrot anthocyanins suppress lipopolysaccharide-induced inflammation in the co-culture of intestinal Caco-2 and macrophage RAW264.7 cells. Food Funct. 2016 Jan;7(1):557-64.

31. Braun-Moscovici Y, Toledano K, Markovits D, Rozin A, Nahir AM, Balbir-Gurman A. Vitamin D level: is it related to disease activity in inflammatory joint disease? Rheumatol Int. 2011 Apr;31(4):493-9.

32. Haque UJ, Bathon JM, Giles JT. Association of vitamin D with cardiometabolic risk factors in rheumatoid arthritis. Arthritis Care Res (Hoboken). 2012 Oct;64(10):1497-504.

33. Welsh P, Peters MJ, McInnes IB, Lems WF, Lips PT, McKellar G, Knox S, Michael Wallace A, Dijkmans BA, Nurmohamed MT, Sat$\operatorname{tar} \mathrm{N}$. Vitamin D deficiency is common in patients with RA and linked to disease activity, but circulating levels are unaffected by TNF $\otimes$ blockade: results from a prospective cohort study. Ann Rheum Dis. 2011 Jun;70(6):1165-7.

34. Varenna M, Manara M, Cantatore FP, Del Puente A, Di Munno O, Malavolta N, Minisola G, Adami S, Sinigaglia L, Rossini M. Determinants and effects of vitamin $D$ supplementation on serum 25-hydroxy-vitamin D levels in patients with rheumatoid arthritis. Clin Exp Rheumatol. 2012 Sep-Oct;30(5):714-9.

35. Weiss ST. Bacterial components plus vitamin D: the ultimate solution to the asthma (autoimmune disease) epidemic? J Allergy Clin Immunol. 2011 May;127(5):1128-30.

Received: 26 February 2021

Accepted: 27, July,2021 